\title{
Comparative DNN Model Analysis for Detection of Various types of Optical Noise
}

This paper was downloaded from TechRxiv (https://www.techrxiv.org).

\section{LICENSE}

CC BY-NC-SA 4.0

SUBMISSION DATE / POSTED DATE

$11-02-2022$ / 28-02-2022

\section{CITATION}

Ghosh, Manthan (2022): Comparative DNN Model Analysis for Detection of Various types of Optical Noise. TechRxiv. Preprint. https://doi.org/10.36227/techrxiv.19160615.v1

DOI

10.36227/techrxiv.19160615.v1 


\title{
Comparative DNN Model Analysis for Detection of Various types of Optical Noise
}

\author{
Manthan Ghosh, Nitish Sinha, Dr.Bishanka Brata Bhowmik,
}

\begin{abstract}
Throughout the next millennium, there will be an ongoing demand for more network capacity. The massively increasing number of users, as well as the duration of each user, and therefore the bandwidth, is a crucial factor. For many years, Internet traffic has grown rapidly. These characteristics have led to the development and, surprisingly, quick adoption of high-capacity optical grid systems on commercial sites. In this paper, we will examine the noises that arise in optical fiber communication. Because of the impact of noise and distortion, linear fiber-optic connections must ensure compliance with physics principles, design standards, and recognized engineering practice. The following forms of noise problems are commonly encountered in optical signal propagation: phase noise, ASE noise, and dispersion. In this case, all three forms of noise are recovered from the optical signal by utilizing variational mode decomposition (VMD) and the Wavelet Transform (WT). In this paper, we compare various deep neural models used to identify optical noise. We also displayed the performance analysis of each model as well as the execution time. The suggested technique outperforms the competition and has the potential to be a contender for automatic noise detection in a fiber communication link.
\end{abstract}

Index Terms-Optical Fiber Communication,Variational mode decomposition(VMD), Wavelet Transform, Deep Neural Network, Optical noise, Automated noise detection(AMD).

\section{INTRODUCTION}

W HILE the modern era begins, the telecommunications industry is undergoing massive development with far-reaching implications. There are several elements that contribute to these shifts. First and foremost, there is an ongoing and unabated need for additional network capacity in the context of Communication Engineering (CE). This urge is sparked by a variety of factors. For many years, Internet traffic has been steadily increasing. There have been substantial variances in growth expectations over the years, with rapid growth predictions tripling in four to six months. Despite the fluctuations, these growth projections are typically considerable, with more recent forecasts of over $50 \%$ per year. Meanwhile, large-scale access technologies such as DSL or cable modems that provide a user with $1 \mathrm{Mb} / \mathrm{s}$ of bandwidth have been widely deployed. In the United States, for example, approximately $55 \%$ of people had broadband connections in their homes in 2008, while only $10 \%$ had access via $28-56 \mathrm{~Kb} / \mathrm{s}$ dial-up [1].

In contrast to traditional speaking traffic, data traffic dominates network traffic. Previously, the reverse was true, and as a result, legacy networks were designed to serve voice

\footnotetext{
The authors are associated with Electronics \& Communication Department, Tripura University, Agartala, Tripura (email:bishankabhowmik@tripurauniv.ac.in).
}

more successfully than information. In today's world, data transmission services are widely available and can provide high-quality service for sensitive and demanding applications such as audio and video. These factors have resulted in the development and, unexpectedly, rapid commercialization of high-capacity optical grids from research centers.

Machine learning, on the other hand, is a rising technology that benefits all sorts of businesses in all industries. There are no restrictions on the applications. Machine learning can help many different sorts of enterprises, from medical to investment banking, aviation to information technology, and advertising to administration, adapt and advance in a more agile manner. We may be capable of screening a hugely structured table and detecting patterns, but computers can screen far larger data sets and discover patterns faster than any person or humangenerated table function thanks to AI and Machine Learning. ML helps any system to gather insights quickly and efficiently, reducing analytical time. As a result, machine learning plays a critical role in organizing and analyzing massive amounts of data.

In this study, we will look into sounds that occur in optical fiber transmission. Due to the effects of noise and distortion, linear fiber-optic connections are responsible for guaranteeing conformity with principles of physics, design standards, and established engineering practice. Here we compare different DNN models performance and accuracy in detecting optical noises.

\section{Methodology}

A fiber-optic link consists of transmitters, fiber cable, and receivers. With the incoming radio frequency signal, the transmitter adjusts the optical laser output. This modular optical signal is converted to the original signal through a fiber-optic link to the receiver. In OFC, there are several sorts of noises arise. In our research, we considered amplified spontaneous emission noise, phase noise, and dispersion. We compute the VMD for each form of noise to recover the noise signal since fiber noise occurs over a large frequency range. After noise separation, the wavelet transform will be used to understand the basic concepts of the noise signal as well as the power associated with it and save this noise output as scalogram images as shown in figure:1. Now this data can be used to train and test the network.

\section{VMD}

The empirical mode decomposition approach allows any difficult data set to be broken down into a finite and frequently small number of intrinsic mode functions, which 


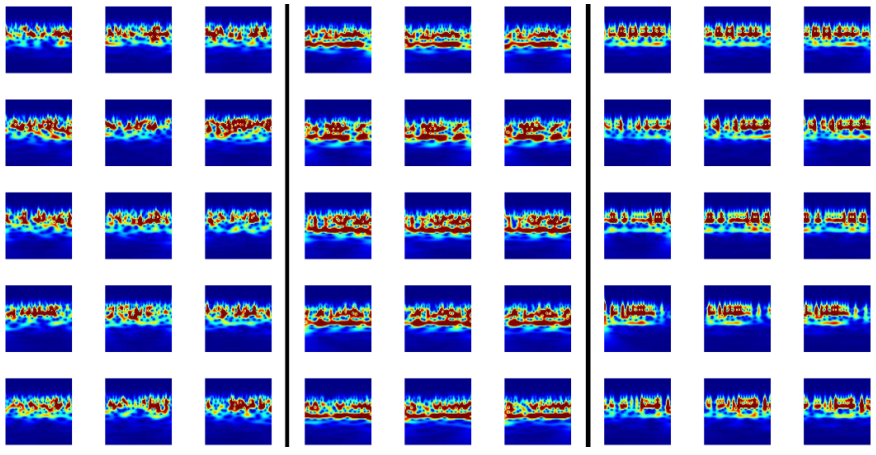

Fig. 1. Wavelet transform of noise signals

allows Hilbert to convert well-conformed. This method of breakdown is adaptive and hence very efficient. Since the breakdown is dependent on the local management of data, it applies to nonlinear and non-stationary operations [2].

In 2014, Dragomiretskiy introduced Variational Mode Decomposition as a novel conscience signal decomposition approach inspired by Huang and Gills [3]. The VMD implies that the signal is the compact support of the Fourier spectrum, like other EMD-like techniques. Based on this assumption, a variable set of Wiener filters is built, which extracts all modes concurrently [4]. The VMD thus works well to resolve closely related modes and reduce the aliasing mode [5]. A clear need for VMD mentioned in Ref. [3] by Dragomiretskiy is that it is necessary to identify the number of modes before VMD is applied. Although it is possible to intentionally detect the number of modes in the frequency domain or after the VMD processing is adapted, Wang in Ref. [6] recently analyzed the filter-bank feature of VMD and various research has been done to improve the VMD adaptability. Most of these improvements are needed to first process the VMD and then optimize variables or integrate the IMFs iteratively [7] [8] [9].

An EMD's undeniable mode is as a signal with a maximum number of local extremes and null crossings. In following research, the concept of modulation criterion was modified somewhat into so-called intrinsic mode functions [2]. The decomposition variable mode (DVM) is an adaptive and almost orthogonal method of decomposition of signals [3]. The method of VMD decomposes the $\mathrm{x}(\mathrm{t})$ signal into a finite number of intrinsic band-limited functions $x_{k}(t)$. VMD incorporates Wiener filtering, Hilbert transformation and analysis, and frequency change through harmonic mixing and the alternating multiplier direction method (ADMM) [3]. This technique provides good precision and reliability for low-frequency spectrum extraction features. It is extensively used in different domains, such as biomedical picture de-noise [10], bearing error detection [11] [12] and pressure fluctuation signal analysis [13]. VMD is a new approach that can degrade a whole signal to a number $\mathrm{K}$ of modes $u_{k}$ out around the central frequency $\omega_{k}$ in such a variable step signal decomposition. VMD is a new approach that can degrade a whole signal to a number $\mathrm{K}$ of modes $u_{k}$ around the central frequency in such a variable step signal decomposition. A limited variational challenge that is defined as follows should be resolved using VMD [14]:

$$
\min _{\left\{u_{k}, \omega_{k}\right\}}\left\{\sum_{k}\left\|\left[\left(\delta(t)+\frac{j}{\pi t}\right) * u_{k}(t)\right] e^{\left(-j \omega_{k} t\right)}\right\|_{2}^{2}\right\}
$$

where $\mathrm{f}$ is really the primary decomposable signal; $u_{k}$ : $\left(u_{1}, u_{2}, u_{k}\right) \omega_{k}:\left(\omega_{1}, \omega_{2}, \omega_{k}\right)$ are abbreviation notes used to indicate the set among all modes and associated center frequencies.

In addressing the restrictions, the penalty term and the Lagrangian multipliers are represented by $\lambda(t)$. These two terms are combined in line with the good convergence characteristics of the finite weight quadratic penalty and the tight implementation by the Lagrangian multiplier of the constraint.

\section{Wavelet Transform}

Wavelet transformations are mathematical techniques that may be used to analyse data with characteristics that change across scales. Frequencies that change over time, transients, and slowly shifting trends are all examples of signal characteristics. Edges and textures are examples of characteristics in pictures. Wavelet transformations were developed to overcome the shortcomings of the Fourier transform. Wavelet analysis is based on decomposing signals into shifted and scaled copies of a wavelet, whereas Fourier analysis is based on decomposing signals into sine waves of specified frequencies. In contrast to a sine wave, a wavelet is a fast-fading, wave-like oscillation. This allows wavelets to represent data at different sizes.

\section{DNN}

Deep learning refers to a type of machine learning techniques that employ many layers to extract higher-level characteristics from raw data [15]. Lower layers in image processing can detect edges, whereas higher layers can identify humanrelevant concepts like numerals, characters, and faces. Artificial neural networks, especially convolution neural networks $(\mathrm{CNN})$, are used in the majority of contemporary deep learning models [16]. Unsupervised learning problems can benefit from deep learning algorithms [17]. This is a significant advantage since unlabeled data is more plentiful than labelled data [18].Deep learning techniques for supervised learning remove feature engineering by converting data into compact intermediate representations similar to main components. A greedy layer-by-layer technique can be used to build deep learning architectures. The objective is to deconstruct these abstractions and determine which characteristics boost performance.

\section{Keras DNN Models}

Keras is a Python interface for ANNs that is available as open-source software. It is a front-end for Tensor-Flow and supports a variety of back ends, such as Microsoft Cognitive Toolkit and others. It is easy to use and extendable, with the objective of facilitating quick DNN research. We are using here all the keras application models to compare the each model's accuracy on the optical data. 
All models used for comparison are listed below:
1) Xception
2) VGG16
3) VGG19
4) ResNet50
5) ResNet101
6) ResNet152
7) ResNet50V2
8) ResNet101V2
9) ResNet152V2
10) Inception $V 3$
11) InceptionResNetV2
12) MobileNet
13) MobileNetV2
14) DenseNet121
15) DenseNet169
16) DenseNet201
17) NASNetMobile
18) NASNetLarge
19) EfficientNetB0
20) EfficientNetB 1
21) EfficientNetB2
22) EfficientNetB3
23) EfficientNetB4
24) EfficientNetB5
25) EfficientNetB6
26) EfficientNetB7

\section{Transfer-Learning}

Transfer-learning (TfL) is basically some the CNN that are designed which is actually giving state of art algorithm to classify different images. It is best first to know about imagenet. Every year a competition is held where in every year people come up with new type of CNN to classify thousand of different types of images and whichever have the highest accuracy get the prize money. In this techniques using the state of art algorithm listed in Table: I and changing the output layer and use that particular algorithm for solving our problem statement. It has become popular in the field of image classification and NLP. The best thing about this method is that it takes less number of iterations and high accuracy rather than a model which build from scratch. Using TfL we can save lot of computation time and resources because many time this pre-trained models are rained on millions of images. If we want to run this type of models on our local system it might take days or even months. All we are doing here is getting all the weights of pre-trained models and then we kindly change the last layer or last few layers sometimes and will get the super high accuracy of our problem statement.

\section{Loss Functions}

To understand how neural network training works, it's important to first understand loss or a cost function, which is what we'll go over in this section. When creating a keras or tensorflow model, we can specify different values for this loss function. We used the "categorical cross entropy"(CCE) loss function here, but other values such as "binary cross
entropy"(BCE), "mean absolute error"(MAE), "mean squared error"(MSE) and so on are also possible. A loss function, also known as a cost function or error function, quantifies the difference between the output and a target value. In our model, we actually output a probability distribution, and from this distribution, we know what the intended target value was from the training data, and then we come up with a method for determining how wrong the model is. There are numerous theoretical approaches we could take. But in general, the loss function of choice for classification that uses softmax as the activation function on the output layer is categorical cross entropy. The formula of CCE is :

$$
L_{i}=-\sum y_{i},{ }_{j} \log \left(\hat{y}_{i},{ }_{j}\right)
$$

$L_{i}$ is sample loss value, $\mathrm{i}$ is $\mathrm{i}$-th sample in a set, $\mathrm{j}$ is label/output index, $\mathrm{y}$ is target value and $\hat{y}$ is predicted value.

The question of why we are using CCE here may arise. The first reason is that when we approach these ML problems from a probabilistic perspective, MSE naturally arises from the linear regression problem and CCE naturally arises from the logistic regression problem. In a multi-classification problem, the MSE does not penalise the model as much as it should for a misclassification, whereas CCE penalises the model heavily for a single misclassification. The gradient of the loss curve is very low in the case of the MSE, so changes in weights are negligible. However, in the case of CCE, the gradient is too steep. As a result, if our model makes a minor error also, it will adjust the weights to train the network more effectively.

\section{MODEL COMPARISON}

In the below figures, all the training and testing model performances have been shown.
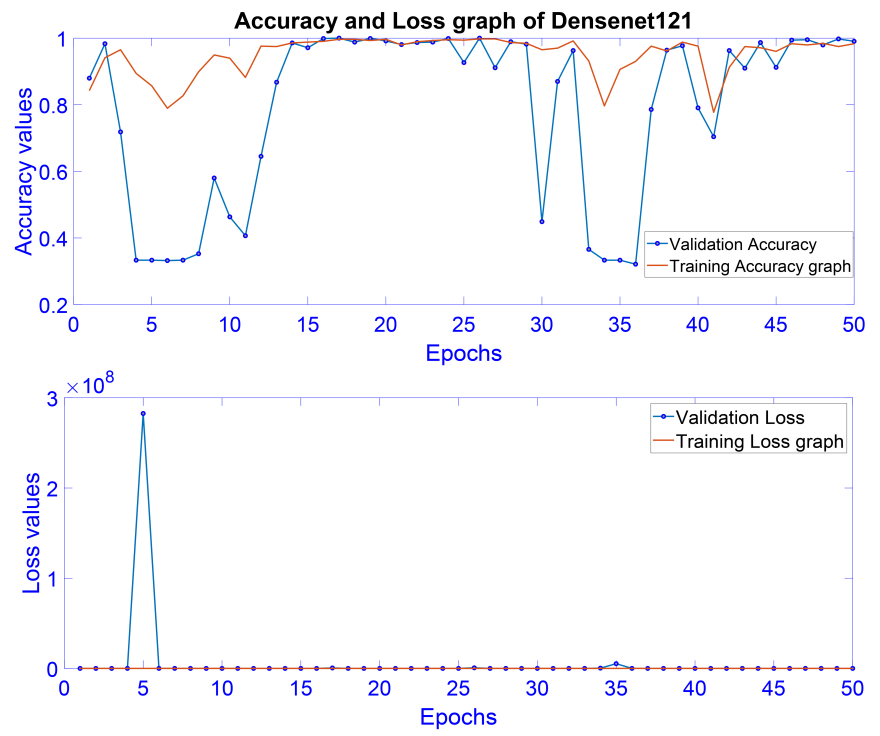

Fig. 2. Densnet121 

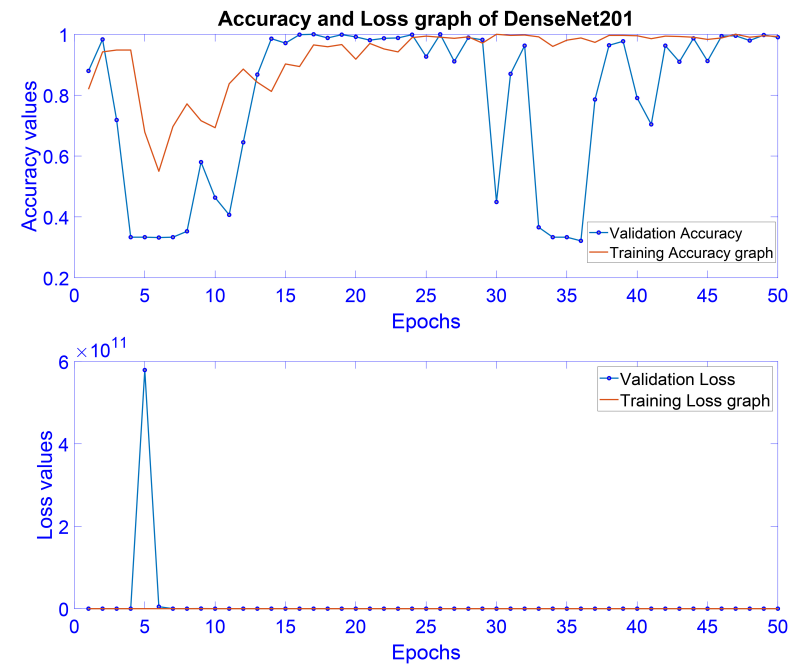

Fig. 3. Densnet201
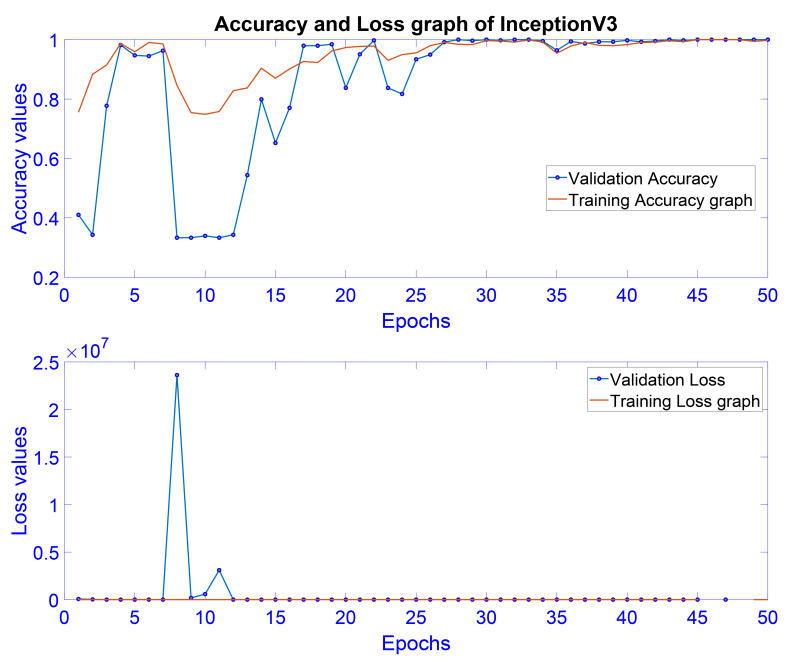

Fig. 4. InceptionV3
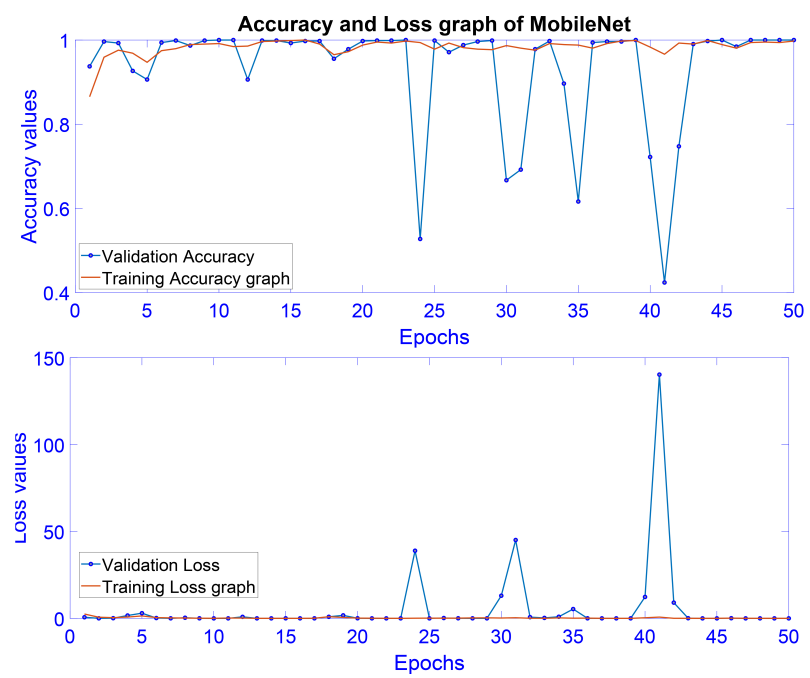

Fig. 5. MobileNet
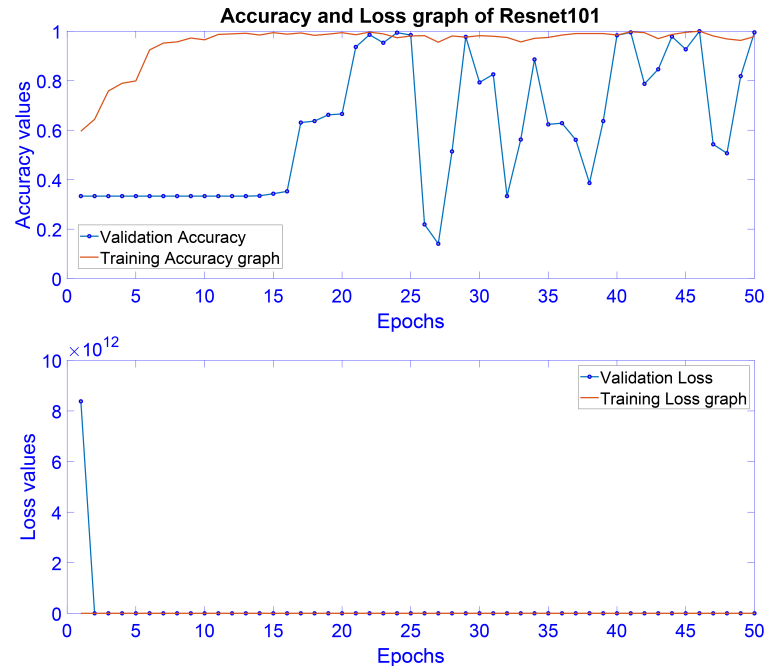

Fig. 6. ResNet101
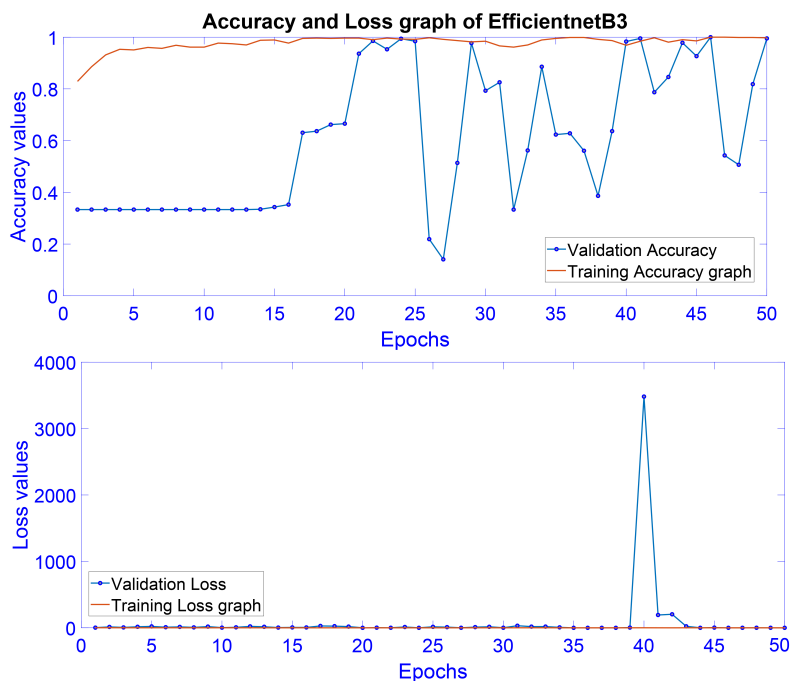

Fig. 7. EfficientNetB3
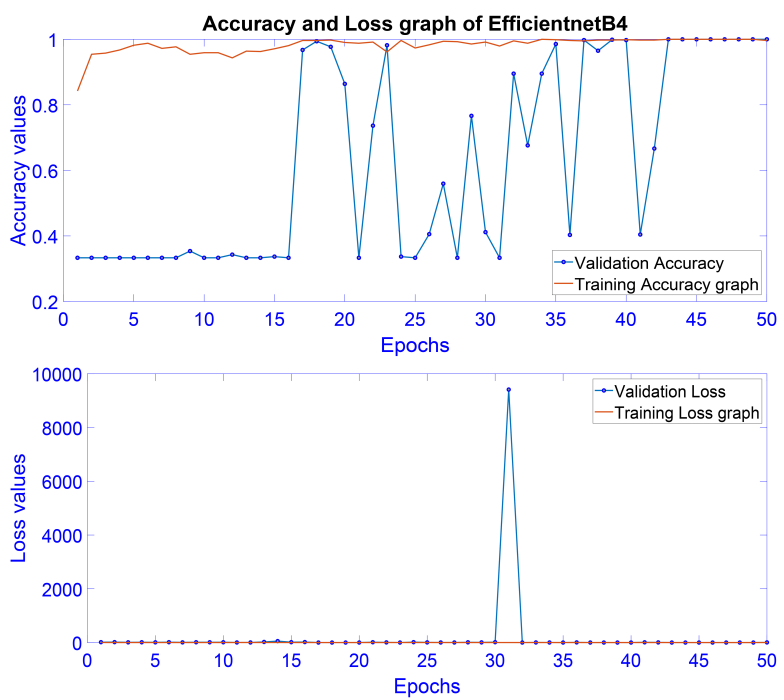

Fig. 8. EfficientNetB4 

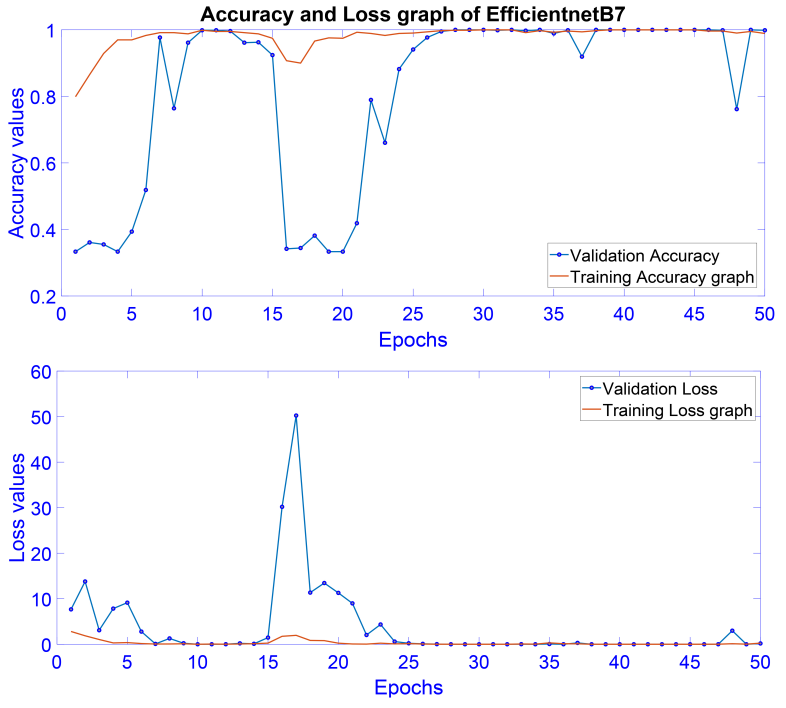

Fig. 9. EfficientNetB7

TABLE I

COMPARISON TABLE

\begin{tabular}{|l|l|l|l|l|}
\hline Model & $\begin{array}{l}\text { Train } \\
\text { Loss }\end{array}$ & $\begin{array}{l}\text { Train } \\
\text { Acc. }\end{array}$ & $\begin{array}{l}\text { Valid } \\
\text { Loss }\end{array}$ & $\begin{array}{l}\text { Valid } \\
\text { Acc } .\end{array}$ \\
\hline Xception & NA & NA & NA & NA \\
\hline VGG16 & 1.0987 & 0.3045 & 1.0986 & 0.3333 \\
\hline VGG19 & 1.0987 & 0.3333 & 1.0986 & 0.3333 \\
\hline ResNet50 & 1.7692 & 0.9627 & $2 \times 10^{4}$ & 0.3394 \\
\hline ResNet101 & 0.0791 & 0.9783 & 0.0145 & 0.9952 \\
\hline ResNet152 & 0.2941 & 0.9266 & 0.5126 & 0.7341 \\
\hline ResNet50V2 & 0.6910 & 0.8183 & 0.6050 & 0.7088 \\
\hline ResNet101V2 & 0.1488 & 0.9458 & 0.0634 & 0.9807 \\
\hline ResNet152V2 & 0.4002 & 0.7978 & 0.3714 & 0.8532 \\
\hline InceptionV3 & 0.0173 & 0.9940 & 1.208 & 1 \\
\hline InceptionResNetV2 & 0.4002 & 0.7978 & 0.3714 & 0.8532 \\
\hline MobileNet & 0.0076 & 0.9976 & 0.0006 & 1 \\
\hline MobileNetV2 & 0.0993 & 0.9615 & 243 & 0.3333 \\
\hline DenseNet121 & 0.0760 & 0.9832 & 0.0240 & 0.9940 \\
\hline DenseNet169 & 0.1167 & 0.9651 & 0.1318 & 0.9519 \\
\hline DenseNet201 & 0.0268 & 0.9928 & 0.0071 & 0.9988 \\
\hline NASNetMobile & 0.0020 & 1 & 201015 & 0.3333 \\
\hline NASNetLarge & 0.0594 & 0.9807 & $2 \times 10^{12}$ & 0.3333 \\
\hline EfficientNetB0 & 0.0405 & 9964 & 10.1856 & 0.3333 \\
\hline EfficientNetB1 & 0.5490 & 0.9723 & 52.6101 & 0.3333 \\
\hline EfficientNetB2 & 0.1103 & 0.9940 & 9.7416 & 0.4838 \\
\hline EfficientNetB3 & 0 & 1 & 0 & 1 \\
\hline EfficientNetB4 & 0.0004 & 1 & 0 & 1 \\
\hline EfficientNetB5 & 0.1636 & 0.9940 & 1.1126 & 0.9110 \\
\hline EfficientNetB6 & 0.0653 & 0.9964 & 0.0993 & 0.9675 \\
\hline EfficientNetB7 & 0.1570 & 0.9988 & 0.0052 & 0.9988 \\
\hline & & & & \\
\hline
\end{tabular}

\section{CONCLUSiON}

As discussed in the paper, ML \& Deep Learning gave optimum performance in the case of noise detection in fiber optic cable. Table: I contains a list of all of the loss and accuracy measures. In our comparative model analysis few models shows the significant high accuracy towards the data we generated. As shown in Table: I the InceptionV3, MobileNet, EfficientNetB3 and EfficientNetB4 models gave the highest accuracy among other models.

\section{ACKNOWLEDGMENTS}

We acknowledge the dept. of Electronics \& Communication Engineering of Tripura University for providing necessary supports.

\section{REFERENCES}

[1] R. Ramaswami, K. Sivarajan, and G. Sasaki, Optical networks: a practical perspective. Morgan Kaufmann, 2009.

[2] N. E. Huang, Z. Shen, S. R. Long, M. C. Wu, H. H. Shih, Q. Zheng, N.C. Yen, C. C. Tung, and H. H. Liu, "The empirical mode decomposition and the hilbert spectrum for nonlinear and non-stationary time series analysis," Proceedings of the Royal Society of London. Series A: mathematical, physical and engineering sciences, vol. 454, no. 1971, pp. 903-995, 1998.

[3] K. Dragomiretskiy and D. Zosso, "Variational mode decomposition," IEEE transactions on signal processing, vol. 62, no. 3, pp. 531-544, 2013.

[4] G. Choi, H.-S. Oh, and D. Kim, "Enhancement of variational mode decomposition with missing values," Signal Processing, vol. 142, pp. 75-86, 2018.

[5] S. Chen, X. Dong, Z. Peng, W. Zhang, and G. Meng, "Nonlinear chirp mode decomposition: A variational method," IEEE Transactions on Signal Processing, vol. 65, no. 22, pp. 6024-6037, 2017.

[6] Y. Wang and R. Markert, "Filter bank property of variational mode decomposition and its applications," Signal Processing, vol. 120, pp. 509-521, 2016.

[7] Z. Li, J. Chen, Y. Zi, and J. Pan, "Independence-oriented vmd to identify fault feature for wheel set bearing fault diagnosis of high speed locomotive," Mechanical systems and signal processing, vol. 85, pp. 512-529, 2017

[8] J. Lian, Z. Liu, H. Wang, and X. Dong, "Adaptive variational mode decomposition method for signal processing based on mode characteristic," Mechanical Systems and Signal Processing, vol. 107, pp. 53-77, 2018.

[9] X. Zhang, Q. Miao, H. Zhang, and L. Wang, "A parameter-adaptive vmd method based on grasshopper optimization algorithm to analyze vibration signals from rotating machinery," Mechanical Systems and Signal Processing, vol. 108, pp. 58-72, 2018.

[10] S. Lahmiri and M. Boukadoum, "Biomedical image denoising using variational mode decomposition," in 2014 IEEE Biomedical Circuits and Systems Conference (BioCAS) Proceedings. IEEE, 2014, pp. 340-343.

[11] K. K. Gupta, K. S. Raju et al., "Bearing fault analysis using variational mode decomposition," in 2014 9th International Conference on Industrial and Information Systems (ICIIS). IEEE, 2014, pp. 1-6.

[12] S. Mohanty, K. K. Gupta, and K. S. Raju, "Comparative study between vmd and emd in bearing fault diagnosis," in 2014 9th International Conference on Industrial and Information Systems (ICIIS). IEEE, 2014, pp. 1-6.

[13] X. An and H. Zeng, "Pressure fluctuation signal analysis of a hydraulic turbine based on variational mode decomposition," Proceedings of the Institution of Mechanical Engineers, Part A: Journal of Power and Energy, vol. 229, no. 8, pp. 978-991, 2015.

[14] W. Ma, S. Yin, C. Jiang, and Y. Zhang, "Variational mode decomposition denoising combined with the hausdorff distance," Review of Scientific Instruments, vol. 88, no. 3, p. 035109, 2017.

[15] L. Deng and D. Yu, "Deep learning: methods and applications," Foundations and trends in signal processing, vol. 7, no. 3-4, pp. 197-387, 2014.

[16] Y. Bengio, Learning deep architectures for AI. Now Publishers Inc, 2009.

[17] Y. Bengio, P. Lamblin, D. Popovici, and H. Larochelle, "Greedy layerwise training of deep networks," in Advances in neural information processing systems, 2007, pp. 153-160.

[18] Y. Bengio, A. Courville, and P. Vincent, "Representation learning: A review and new perspectives," IEEE transactions on pattern analysis and machine intelligence, vol. 35, no. 8, pp. 1798-1828, 2013. 